\title{
CGH Compressed and Transmitted and Reconstructed System with JPEG Baseline Processing and Fresnel Transforming Technique
}

\author{
Non-member Guanglin Yang (Osaka City University) \\ Member Eiji Shimizu (Osaka City University)
}

\begin{abstract}
A new CGH (Computer Generated Hologram) compressed and transmitted and reconstructed system (CCTRS) has been established in which JPEG baseline-encoding model and Fresnel transforming technique (FTT) have been adapted. This system can be applied in the remote signal processing using the digital filter of CGH. When the compression ratio is achieved to $1.5303 \%$, the information of processed CGH can be effectively reconstructed using FTT. Moreover, in this system the size of image displayed is bigger (i.e., $\left.18.07 \times 18.07\left(\mathrm{~cm}^{2}\right)\right)$, the system structure is simpler, the system costs lower than that using the electron holographic hardware display system, and can be widely applied by computer operators. In experiments, in terms of the holographic principle of E.N.Leith and J.Upatnieks, a CGH has been made with a computer, the information of processed CGH has been analyzed and the information distribution of processed CGH has been compared with the one of the original CGH. The reconstructed image quality of processed CGH has been discussed. Finally, Compression ratio (R), Mean squared error (MSE) and Peak signal to noise ratio (PSNR) have been precisely calculated and analyzed to evaluate the image quality of processed CGH and the reconstructed image quality of processed CGH. And the noise influence of the system for processed CGH has been analyzed and discussed in detail. In CGH compressed and transmitted, the reason has been carefully explained why the error problems can be caused as the pixel's amplitude and phase information of CGH varied. This method of processing CGH has been effectively verified by experiments.
\end{abstract}

Keywords: CCTRS, CGH, JPEG, FTT.

\section{Introduction}

It is well known that a CGH contains a lot of information. How to quickly transmit such huge information exists some problems in the electronic system (e.g., the internet). In this paper, we will discuss a few problems when CGH is compressed and transmitted and reconstructed. JPEG (joint photographic experts group) baseline encoding technique ${ }^{(1 \sim 4)}$ and FTT ${ }^{(14,15)}$ have mainly been adapted to process CGH. And a CGH compressed and transmitted and reconstructed system has been established as shown in Fig.1.

In researching, some documents ${ }^{(7 \sim 13,15)}$ about the digital hologram processed have been investigated. Their researching is about the electron holographic hardware display system. However, at present the computer has been quickly developed and applied in every field, CGH has the potential application, thus we have been adapted only the software system to complete the total process in which CGH (i.e., 2-D digital hologram) is compressed as much as possible and transmitted quickly and reconstructed effectively.

$\mathrm{CGH}$ is a term used to refer to a hologram with a computer. And it can be described as a mathematical function of a wave front or an object represented by an array of points ${ }^{(6 \sim 8)}$. Therefore, the computer can calculate the amplitude and phase transmittance of the hologram. Consequently, using a computer we can create the optical elements (e.g., the digital filters) that cannot be fabricated by conventional methods ${ }^{(7,8)}$. So $\mathrm{CGH}$ has some special instinctive advantages.

$\mathrm{CGH}$ is a collection of special optical element (i.e., pixel). CGH also is a matrix of picture pixels ${ }^{(7,8)}$ Each pixel contains information about its grayscale. But its difference (i.e., comparing with the general image) is that $\mathrm{CGH}$ is an interference fringe, the pixels of CGH interfere with each other so as to produce diffraction. According to this characteristic, we are able to exploit the inherent redundancy information of CGH. In compression, the pixel's size of CGH has been adjusted before CGH made, and then the image encoding and quantization technique have been applied to reduce the diffraction boundaries range of contiguous pixels, because $\mathrm{CGH}$ has enough redundant information (i.e., a part of the information given by a pixel can be found in the neighbor pixels $)^{(9 \sim 15)}$.

In processing, if the lost information of CGH exceeds certain limit value, its original information distribution (i.e., the amplitude and phase functions) will be varied. In terms of the Detour Phase Holograms principle of B. R. Brown and A. W. Lohmann, we can understand, the size variation of CGH pixels is determined by amplitude function, the position variation of CGH pixels is determined by phase function. Thus in com- 


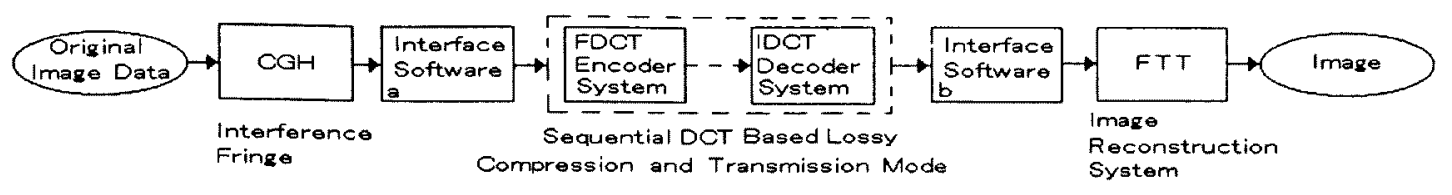

Fig. 1. CCTRS using JPEG baseline processing technique and FTT.

pression and transmission, if the pixel's size can be reduced (i.e., the limit value of $\mathrm{CGH}$ compressed can't be exceeded), and the pixel's position can be restored after CGH compressed, the image of CGH can be effectively compressed and transmitted and reconstructed.

Through adapting JPEG baseline encoding model, we have found, the compression ratio can be improved by reducing the redundant information contained in the original CGH. Moreover some of the smallest tones of CGH cannot be restored by the decoder step. This information can be deleted without degrading the quality of the CGH. We can loss some of the smallest details of CGH, but we can spare a lot of bytes. Thus if the information content of CGH can be reduced, CGH can be quickly transmitted and effectively reconstructed in the electron system.

According to above ideas, a sequential DCT (Discrete Cosine Transform) based loss compression mode of JPEG family ${ }^{(1,2,5)}$ has been adapted to process the CGH. Using this mode, the storage and transmission cost for CGH can be dramatically reduced. And using this system, we have made some experiments for processing $\mathrm{CGH}$, compared theirs specialties of the processed CGH with the one of the original CGH, and analyzed the noise influence of the transmitted CGH in CCTRS. At last, its advantages and disadvantages of this processing method have been analyzed and discussed.

In Sect. 1 the system structure of CCTRS and background of researching $\mathrm{CGH}$ is described. In Sect. 2 the principle of CGH is described. In Sect. 3 JPEG baseline model is described. In Sect. 4 the image reconstructed algorithm of CGH is described. In Sect.5 the software interface is described. In Sect.6 and Sect.7, the experiment analyses, discussion and the conclusion are respectively described.

\section{Principle of Computer-Generated Holo- gram}

According to the theory of E. N. Leith and J. Upatnieks in off-axis reference beam holograms ${ }^{(7)}$. we have generated an on-axis reference beam interference hologram of an oblique point line with a computer. That is, the amplitude and phase transmittance of a hologram recorded under ideal conditions is

$$
\begin{aligned}
I(x, y)= & {[r(x, y)+a(x, y)][r(x, y)+a(x, y)]^{*} } \\
= & \mid R(x, y) \exp [j \phi(x, y)] \\
& +\left.A(x, y) \exp [j \psi(x, y)]\right|^{2} \\
= & R(x, y)^{2}+A(x, y)^{2}+2 R(x, y) A(x, y) \\
& \cdot \cos [\phi(x, y)-\psi(x, y)] . \cdots \cdots \cdots
\end{aligned}
$$

Table 1. Experiment data of an oblique point line about a CGH.

\begin{tabular}{c|c}
\hline wave - length $(\lambda)$ & $0.6328 \mu \mathrm{m}$ \\
\hline sampling - points & 21 \\
\hline$L_{x} \times L_{y}$ & $512 \times 512$ \\
\hline pixel $_{x} \times$ pixel $_{y}$ & $0.025 \times 0.025 \mathrm{~mm}^{2}$ \\
\hline
\end{tabular}

In Eq.(1), $r(x, y)=R(x, y) \exp (j \phi(x, y))$ represents the tilted reference wave and $a(x, y)=$ $A(x, y) \exp (j \psi(x, y))$ is the object wave. $I(x, y)$ is the resulting intensity variation of the interference pattern between the object wave and the reference wave. In experiment we have chosen that the positions of the reference beam $r(x, y, z)$ and the object wave $a(x, y, z)$ are respectively $r(0,0,1500(\mathrm{~mm}))$ and $a(0,0,500(\mathrm{~mm}))$.

In terms of Eq.(1) and Table 1, a CGH of an oblique point line and its histogram have been made by a computer as shown in Fig.2, 10. Using FTT, the reconstructed original image of CGH has been shown in Fig.7.

\section{JPEG baseline Processing Model}

3.1 Requirements of JPEG Processing Image In JPEG baseline processing, the images are compressed with a single component. In practice, images may be represented by multiple color components, each at a different resolution. However in this paper, because CGH is a grayscale interference fringe, the problem of multiple color components doesn't exist. And JPEG sets no restrictions on the type of the input grayscale or color space. Instead, it views each image as a collection of image components. The maximum number of color or grayscale components in JPEG is 255. Each component consists of a rectangular array of samples (pixels). Each sample may be represented by $p$-bits of precision. In JPEG, $p$ can be either 8 or 12 for DCT-based coders and from 2 to 16 for lossless coders. The images with other pixel resolutions can still be coded using JPEG. However, pixel values have to be shifted to be within the resolutions supported by JPEG. Therefore, it is not necessary that all color or grayscale components have the same dimensions. JPEG's aim for a generic compression standard requires its proposal to accommodate a variety of source image formats ${ }^{(1,2,3)}$.

3.2 JPEG Baseline Algorithm The JPEG baseline algorithm is a DCT based compression algorithm. It is the simplest algorithm among the JPEG DCT based algorithms. For all other DCT-based JPEG algorithms, the JPEG specification requires that the baseline decoding process must present in the decoders to provide a default decoding capability. For the information compressed and transmitted of CGH, JPEG 
baseline can suit the requirement of the image reconstructed of CGH. This is one of the reasons that JPEG baseline is adapted to process the information of $\mathrm{CGH}$. The JPEG baseline algorithm is the only one that has been widely used among the JPEG family. And it is appropriate for coding continuous-tone images containing from 1 to 4 (typically color) components.

The JPEG baseline system is described in Ref.(1). The encoder diagram contains an $8 \times 8$ FDCT (forward discrete cosine transform), a quantizer, and a Huffman encoder. In the encoding process, samples of the input component of a digital image are divided into $8 \times 8$ blocks. Each block has 64 samples. By applying FDCT to each block, 64 values in the DCT domain can be obtained. One of them is the DC coefficient (zero frequency coefficients). Other 63 values are $\mathrm{AC}$ coefficient (nonzero frequency coefficient). After reordering these coefficients, they are quantized, and then encoded by a Huffman encoder. In Ref.(1), Quantization and Huffman encoding make use of table look-up techniques. The decoder has a Huffman decoder, a dequantizer, and an $8 \times 8$ IDCT (inverse discrete cosine transform). By inverting the encoding process, a reconstructed $8 \times 8$ block of samples can be obtained. This model detailed in Refs. $(1), \sim(5)$.

The JPEG baseline implementation uses Huffman coding only. It also restricts the number of Huffman tables to four: two for the $\mathrm{AC}$ components and two for the DC components. This is not a major constraint, since most applications operate on luminance-chrominance data and uses one set of Huffman tables for the luminance component and one set of tables for chrominance components. This restriction can also be bypassed if one uses noninterleaved data. In that case, a new set of tables may be loaded before the decompression of each color component. Details for the baseline Huffman coder are presented in Refs.(3),(4).

\section{Image Reconstructed of CGH with FTT}

We can know, the eye can't recognize the image information of $\mathrm{CGH}$ processed, its image information only can be observed under the optical Fourier transform lens system. Therefore, according to the Fourier transform method of optical holography, we have established this program system to imitate this optical Fourier transform. It is one of the reasons why Fresnel transform algorithm is adapted in this system.

In FTT, the object's diffraction wave $h\left(x_{2}, y_{2}\right)$ which is recorded on the hologram plane is the Fresnel transforming function of the object's amplitude distribution $h\left(x_{1}, y_{1}\right)$ in the distance $z$. Therefore, it can be expressed as follow:

$$
\begin{aligned}
h\left(x_{2}, y_{2}\right)= & \frac{j k}{2 \pi} \frac{\exp (-j k z)}{z} \int_{-\infty}^{+\infty} \int_{-\infty}^{+\infty} h\left(x_{1}, y_{1}\right) \\
& \cdot \exp \left[-\frac{j k}{2 z}\left(\left(x-x_{1}\right)^{2}+\left(y-y_{1}\right)^{2}\right)\right] \\
& d x_{1} d y_{1} \ldots \ldots \ldots \ldots \ldots \ldots \ldots
\end{aligned}
$$

where $k$ is the wave number $2 \pi / \lambda$.
Because the object's diffraction wave recorded is the Fresnel diffraction in $z$, if the plane or spherical reference wave would be adapted to illuminate the hologram plane, then the original image of object can be reconstructed in the distance $z^{(17)}$.

In light of this theory, we have designed the image reconstruction system of CGH with Fresnel transform algorithm. In image reconstruction of $\mathrm{CGH}$, according to the integral evaluation of Eq.(2) the images can be obtained with a computer. Eq.(2) is a Fresnel transforming function of the hologram $h\left(x_{1}, y_{1}\right)$. This equation is a convolution of the hologram $h\left(x_{1}, y_{1}\right)$ and the propagation function $p(x, y)$, that is

$$
h\left(x_{2}, y_{2}\right)=h\left(x_{1}, y_{1}\right) * p\left(x_{1}, y_{1}\right)
$$

Where $*$ is a convolution. According to the convolution theory, Eq. (3) can be derived from the inverse Fourier transform of the Fourier transforms of two functions

$$
h\left(x_{2}, y_{2}\right)=F^{-1}\left[F\left[h\left(x_{1}, y_{1}\right)\right] \cdot F\left[p\left(x_{1}, y_{1}\right)\right]\right]
$$

The propagation function $p(x, y)$ can be written by Eq.(2)

$$
p(x, y)=\exp \left[-\frac{j \pi}{\lambda z}\left(x^{2}+y^{2}\right)\right] .
$$

The propagation function $p(x, y)$ can be analyzed by the Fourier transform

$$
\begin{aligned}
P(\xi, \eta) & =F[p(x, y)] \\
& =\exp \left[j \pi \lambda z\left(\xi^{2}+\eta^{2}\right)\right]
\end{aligned}
$$

Therefore, in the coordinates $\xi$ and $\eta$ of the Fourier transform plane, the Fresnel transform of the function $h\left(x_{2}, y_{2}\right)$ is one time Fourier transform and one time inverse Fourier transform of the function $h\left(x_{1}, y_{1}\right)$ and $p\left(x_{1}, y_{1}\right)$.

In Fresnel transform calculating, FFT (Fast Fourier Transform) of Cooley and Tukey is used. Assuming that the compressed CGH is divided into meshes of $N \times N$ cells where each cell as a square of $\Delta_{x} \times \Delta_{y}$ and each cell is considered as one sampling point, the sampled hologram is represented as follows:

$$
\begin{aligned}
h\left(N x_{1}, N y_{1}\right)= & \sum_{n=0}^{N-1} \sum_{m=0}^{N-1} h(x, y) \\
& \cdot \delta\left(\frac{x}{\Delta_{x}}-m\right) \delta\left(\frac{y}{\Delta_{y}}-n\right) \cdots .
\end{aligned}
$$

Where $m$ and $n$ are positive integers including zero and $\delta$ is a delta function. The Fourier transform $H(\xi, \eta)$ of the sampled hologram of Eq.(7) is represented as follows:

$$
\begin{aligned}
H(\xi, \eta)= & \int_{-\infty}^{+\infty} \int_{-\infty}^{+\infty} \sum_{n=0}^{N-1} \sum_{m=0}^{N-1} h(x, y) \delta\left(\frac{x}{\Delta_{x}}-m\right) \\
& \cdot \delta\left(\frac{y}{\Delta_{y}}-n\right) \exp [-j 2 \pi(\xi x+\eta y)] d x d y \\
= & \Delta_{x} \Delta_{y} \sum_{n=0}^{N-1} \sum_{m=0}^{N-1} h\left(m \Delta_{x}, n \Delta_{y}\right) \\
& \cdot \exp \left[-j 2 \pi\left(\frac{m L_{x} \xi}{\left(L_{x} / \Delta_{x}\right)}+\frac{n L_{y} \eta}{\left(L_{y} / \Delta_{y}\right)}\right)\right]
\end{aligned}
$$


Where $L_{x}=N \Delta_{x}$ and $L_{y}=N \Delta_{y}$ are the lengths of the sides of the reduced CGH. Assuming that $H(\xi, \eta)$ of Eq. (8) is sampled at intervals $1 / L_{x}$ and $1 / L_{y}$ with respect to the coordinates $\xi$ and $\eta$ on the Fourier transform plane, Eq.(8) can be rewritten as follow:

$$
\begin{aligned}
H(\dot{m}, \dot{n})= & \Delta_{x} \Delta_{y} \sum_{n=0}^{N-1} \sum_{m=0}^{N-1} h\left(m \Delta_{x}, n \Delta_{y}\right) \\
& \cdot \exp \left[-\frac{j 2 \pi}{N}(m \dot{m}+n \dot{n})\right] \cdots \cdots
\end{aligned}
$$

where $\dot{m}$ and $\dot{n}$ are positive integers including zero and $H\left(\dot{m} / L_{x}, \dot{n} / L_{y}\right)$ is expressed by $H(\dot{m}, \dot{n})$. The digital Fourier transform $H(\dot{m}, n)$ can be calculated from the samples values $h\left(m \Delta_{x}, n \Delta_{y}\right)$ of original analog hologram according to Eq.(9) by the FFT method.

The Fourier transform $P(\xi, \eta)$ of the propagation function $p(x, y)$ can be derived analytically and the Fourier transform thus obtained is given by Eq.(10).

$$
\begin{aligned}
P(\xi, \eta)= & \int_{-\infty}^{+\infty} \int_{-\infty}^{+\infty} \exp \left[-\frac{j \pi}{\lambda z}\left(x^{2}+y^{2}\right)\right. \\
& -j 2 \pi(\xi x+\eta y)] d x d y \\
= & -j \lambda z \exp \left[\frac{j \pi \lambda z}{L^{2}}\left(L^{2} \xi^{2}+L^{2} \eta^{2}\right)\right] .
\end{aligned}
$$

where $L$ is the length of one side of the reduced CGH which is assumed to be a square of $L \times L$ for simplicity of analysis. To make the coordinates $(\xi, \eta)$ on the Fourier transform plane in Eq.(10) coincident with the number coordinates $(\dot{m}, \dot{n})$ in Eq. $(9), P(\xi, \eta)$ is sampled at intervals $1 / L\left(=1 / L_{x}=1 / L_{y}\right)$ and rewritten as follows:

$$
P(\dot{m}, \dot{n})=-j \lambda z \exp \left[j \pi \lambda z\left(\dot{m}^{2}+\dot{n}^{2}\right)\right]
$$

where $P(\dot{m} / L, \dot{n} / L)$ is expressed by $P(\dot{m}, \dot{n})$. The Fourier transform is expressed as follows:

$$
P(\dot{m} / L, \dot{n} / L)=-j \lambda z \exp \left[\frac{j \pi \lambda z}{L^{2}}\left(\dot{m^{2}}+\dot{n^{2}}\right)\right]
$$

where $L$ is the length of one side of the CGH which is assumed to be a square of $L \times L$ for simplicity of analysis. The coefficient $\rho$ in Eq.(12) is expressed as follows:

$$
\rho=\frac{\pi \lambda z}{L^{2}}
$$

Eq.(13) indicates that the position of image reconstructed plane can be expressed in terms of CGH's parameters. If the distance $z$, the wavelength $\lambda$ and the dimension $L^{2}$ of the CGH plane are known, we can calculate the $\rho$ value ${ }^{(16,17)}$.

Therefore, according to these parameters and Eq.(4), the original image of CGH can be reconstructed with a computer. The processing steps of CGH reconstructed are: (1) digitizing and sampling $\mathrm{CGH} h\left(x_{1}, y_{1}\right)$; (2) calculating the digital Fourier transform $H(\dot{m}, \dot{n})$ from $\mathrm{CGH}$; (3) analyzing and deriving the Fourier transforming $P(\xi, \eta)$ of the propagation function $p(x, y) ;(4)$ calculating the values of the Fourier transform $P(\dot{m}, \dot{n})$ at each number coordinate; (5) calculating the $H(\dot{m}, \dot{n})$ $P(\dot{m}, \dot{n})$ of both Fourier transform; (6) calculating the inverse digital Fourier transform of $H \cdot P$ corresponding to the image $O\left(x_{2}, y_{2}\right)$ of the original object.

\section{Software Interface}

In CCTRS, because we have made the CGH that it is a binary code fringe, its format must be converted as the image format of "pgm" to match JPEG baseline system. Thus, the interface program has been adapted to convert it. Moreover, the output image of JPEG baseline system must be converted to match the FTT system. In experiment, the converted CGH can be directly reconstructed using $\mathrm{FTT}$, and none of influence exists in conversion experiment (Note: in converted format, $\mathrm{CGH}$ is a raw grayscale image, its byte header is "0".).

\section{Experiment Analyses and Discussion}

The experiment results of CCTRS have been detailed in Table $2 \sim 4$ and Fig. $2 \sim 14$.

In Table 2, Experiment data of the original, compressed, decompressed CGH and $R \%$ has been described. The experiments are about the original, compressed, decompressed, reconstructed images of CGH and the histograms of processed CGH as shown in Fig. $2 \sim 12$ (Note: the reconstructed image size of CGH is $L_{x} \times L_{y}=1024 \times 1024$, the size of CGH is $L_{x} \times L_{y}=512 \times 512$, because FTT is a spherical wave transforming algorithm.).

In Table 3, Relationship data of $R \%, M S E$ and $P S N R$ about comparing the original CGH with the decompressed CGHs in CCTRS have been described. $q(N)$ is defined as quality $N$ of processed image. The experiment is about the distortion measure of processed CGH as shown in Fig.13.

In Table 4, Relationship data of $R \%, M S E$ and $P S N R$ about comparing the reconstructed image of the original $\mathrm{CGH}$ with the reconstructed images of processed CGHs using FTT have been described in CCTRS. The experiment is about the distortion measure of the reconstructed image quality of processed CGHs as shown in Fig.14.

6.1 Noise Influences of FDCT / IDCT In JPEG baseline processing, the key processing steps are the DCT-based modes. We think not to be enough pre-

Table 2. Experiment data of the original, compressed and decompressed CGH in JPEG baseline processing.

\begin{tabular}{c|c|c|c}
\hline$q(N)=2$ & $C G H_{\text {original }}$ & $C G H_{\text {compressed }}$ & $C G H_{\text {decompressed }}$ \\
\hline size & $257 \mathrm{kB}$ & $4 k \mathrm{k}$ & $257 \mathrm{kB}$ \\
\hline$R(\%)$ & & 1.5303 & 100 \\
\hline$q(N)=3$ & $C G H_{\text {original }}$ & $C G H_{\text {compressed }}$ & $C G H_{\text {decompressed }}$ \\
\hline size & $257 \mathrm{kB}$ & $6 \mathrm{kB}$ & $257 \mathrm{kB}$ \\
\hline$R(\%)$ & & 2.0971 & 100 \\
\hline$q(N)=5$ & $C G H_{\text {original }}$ & $C G H_{\text {compressed }}$ & $C G H_{\text {decompressed }}$ \\
\hline size & $257 \mathrm{kB}$ & $11 \mathrm{kB}$ & $257 \mathrm{kB}$ \\
\hline$R(\%)$ & & 3.9936 & 100 \\
\hline$q(N)=10$ & $C G H_{\text {original }}$ & $C G H_{\text {compressed }}$ & $C G H_{\text {decompressed }}$ \\
\hline size & $257 \mathrm{kB}$ & $23 \mathrm{kB}$ & $257 \mathrm{kB}$ \\
\hline$R(\%)$ & & 8.6722 & 100 \\
\hline
\end{tabular}




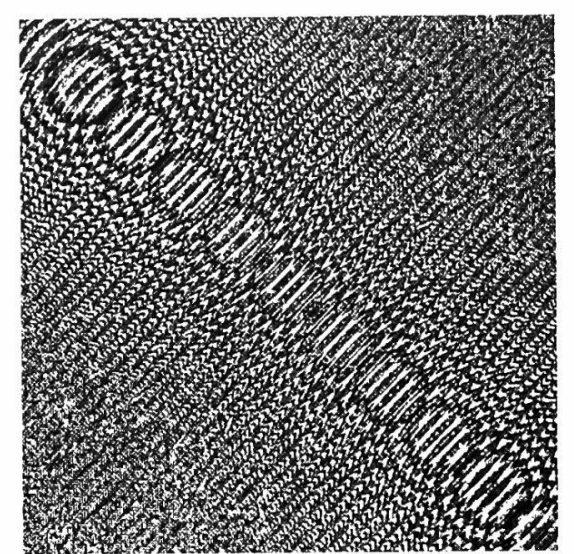

Fig. 2. The original $\mathrm{CGH}$ in on-axis reference beam (size: $257 \mathrm{~kb}, 18.07 \times 18.07 \mathrm{~cm}^{2}$, $512 \times 512$ pixel $^{2}, 8 \mathrm{bpp} /$ grayscale.).

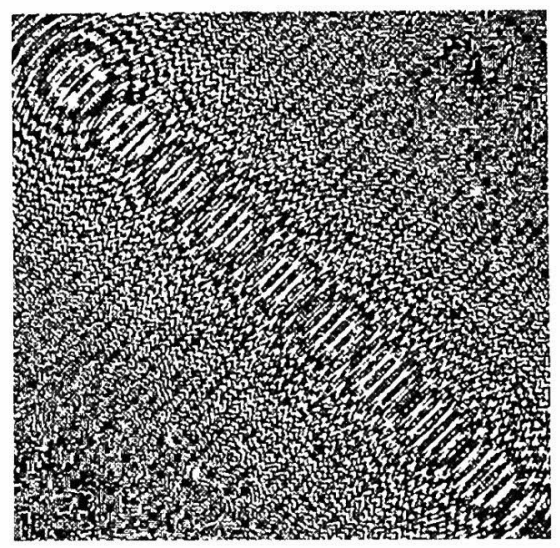

Fig. 3. The compressed CGH using JPEG baseline encoding system (size: $23 \mathrm{~kb}, 18.07 \times 18.07 \mathrm{~cm}^{2}$, $512 \times 512$ pixel $^{2}, 8 \mathrm{bpp} /$ grayscale, $\mathrm{q}(\mathrm{N})=10, R \%=8.95 \%$.).

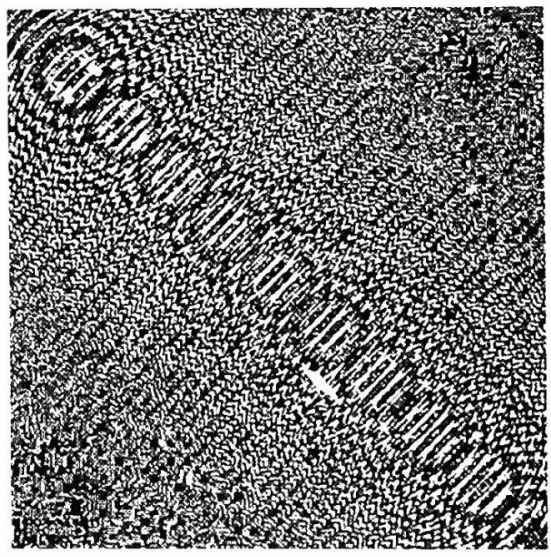

Fig. 4. The decompressed CGH using JPEG baseline decoding system (size: $257 \mathrm{~kb}, 18.07 \times 18.07 \mathrm{~cm}^{2}$ $512 \times 512 \mathrm{pixel}^{2}, 8 \mathrm{bpp} /$ grayscale, $\mathrm{q}(\mathrm{N})=10, R \%=100 \%$.).

cision that a $\mathrm{CGH}$ is divided into $8 \times 8$ blocks and Baseline JPEG stores images with 8 bits per color sample. If JPEG processes CGH using more bits per pixel sam-

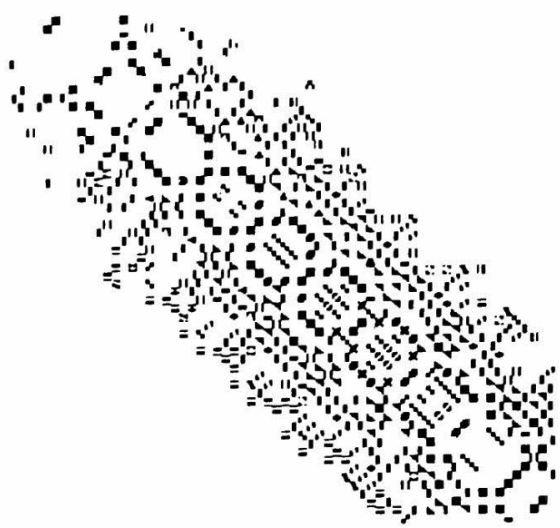

Fig. 5. The compressed CGH using JPEG baseline encoding system (size: $4 \mathrm{~kb}, 18.07 \times 18.07 \mathrm{~cm}^{2}$ $512 \times 512$ pixel $^{2}, 8 \mathrm{bpp} /$ grayscale, $\mathrm{q}(\mathrm{N})=2, R \%=1.5303 \%$.)

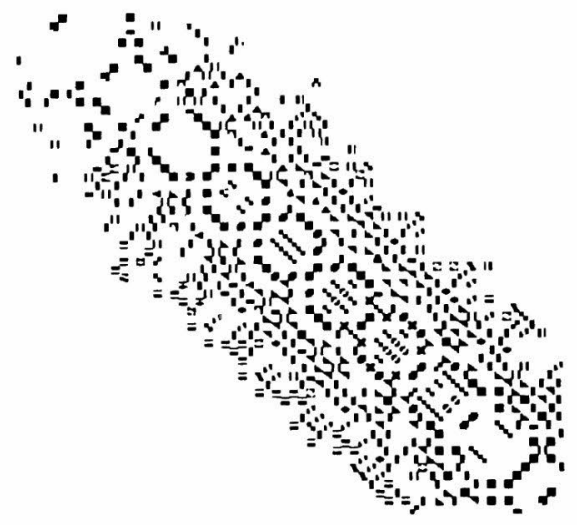

Fig. 6. The decompressed CGH using JPEG baseline decoding system (size: $257 \mathrm{~kb}, 18.07 \times 18.07 \mathrm{~cm}^{2}$, $512 \times 512$ pixel $^{2}, 8 \mathrm{bpp} /$ grayscale, $\mathrm{q}(\mathrm{N})=2, R \%=100 \%$.).

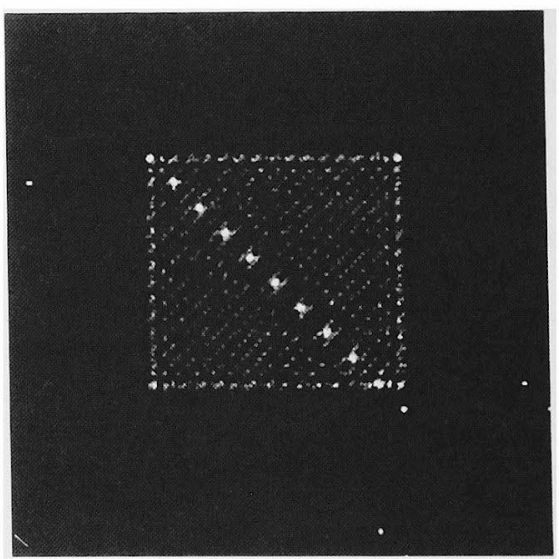

Fig. 7. The reconstructed image of original $\mathrm{CGH}$ using FTT (size: $1024 \mathrm{~kb}, 36.13 \times 36.13 \mathrm{~cm}^{2}$, $1024 \times 1024$ pixel $^{2}, 8 \mathrm{bpp} / 256$.).

ples, the accuracy of the compressed and transmitted CGH can be improved in CCTRS. In principle, DCT introduces no loss to the source image samples. It merely 


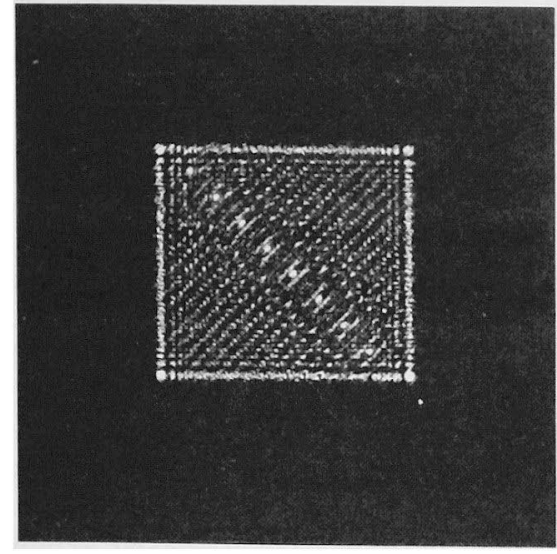

Fig. 8. The reconstructed image of the decompressed CGH using FTT (size: $1024 \mathrm{~kb}$, $36.13 \times 36.13 \mathrm{~cm}^{2}, 1024 \times 1024$ pixel $^{2}, 8 \mathrm{bpp} / 256$, $\mathrm{q}(\mathrm{N})=10, R \%=8.6733 \%$.)

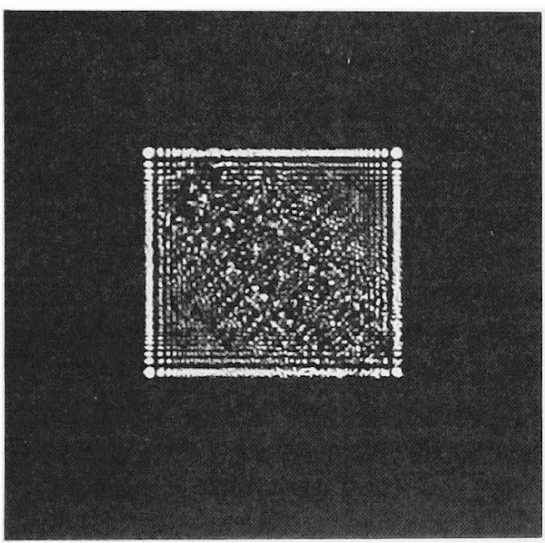

Fig. 9. The reconstructed image of the decompressed CGH using FTT (size: $1024 \mathrm{~kb}$, $36.13 \times 36.13 \mathrm{~cm}^{2}, 1024 \times 1024 \mathrm{pixel}^{2}, 8 \mathrm{bpp} / 256$, $\mathrm{q}(\mathrm{N})=2, R \%=1.5303 \%$.).

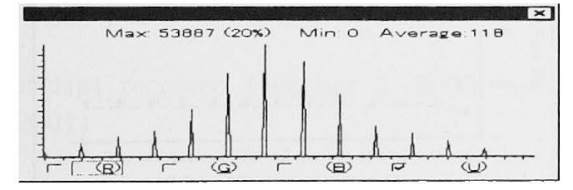

Fig. 10. A histogram of the original CGH.

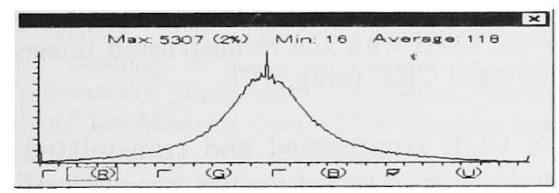

Fig. 11. A histogram of the compressed and decompressed CGH as $\mathrm{q}(\mathrm{N})=10, R \%=8.6733 \%$.

transforms them to a domain in which they can be more efficiently encoded. However, in practical, FDCT and IDCT implementations are only satisfied with the precise requirements of JPEG standard. And no physical implementation can compute them with perfect accu-

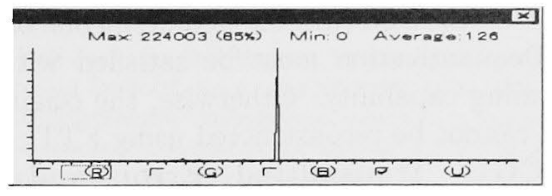

Fig. 12. A histogram of the compressed and decompressed $\mathrm{CGH}$ as $\mathrm{q}(\mathrm{N})=2, R \%=1.5303 \%$.

racy. Because of DCT relationship to the DFT, many different algorithms with FDCT and IDCT are only approximately computed. Indeed, research in fast DCT algorithms is ongoing and no single algorithm is optimal for all implementations.

Even in light of the finite precision of the DCT in puts and outputs, independently designed implementations of the very same FDCT or IDCT algorithm which differ even minutely in the precision by which they represent cosine terms or intermediate results, or in the way they sum and round fractional values, will eventually produce slightly different outputs from identical inputs.

And in JPEG proposed standard, neither a unique FDCT algorithm nor a unique IDCT algorithm. This makes compliance somewhat more difficult to confirm, because two compliant encoders (or decoders) generally will not produce identical outputs given identical inputs. Therefore, this algorithm model is only to ensure against crudely inaccurate cosine basis functions that would degrade image quality.

In JPEG, the compressed file contains an approximation of the original image since the high spatial frequency information has been removed. To reproduce the data compressed. The IDCT is the process of approximated original image. Therefore, the spatial pixel data representation of the given image is not the original image data. Therefore, these will produce some influences for $\mathrm{CGH}$ reconstructed.

6.2 Noise Influences of Quantization / Dequantization The quantization step is used to reduce the magnitude of DCT coefficients and to increase the number of zero value coefficients based on the eye's ability to detect different levels at a given frequency. The values are chosen to match the sensitivity of the eye. Small quantization values are chosen for low frequency and higher values for high frequency coefficients. The JPEG baseline model is considered a "lossy" compressor because the reconstructed image is not identical to the original. Therefore, using Fresnel transform algorithm, the noise can be brought in the reconstructed image. If it is lossless coders or the compressed CGH can be controlled in limit value, that creates images identical to the original, achieve inferior compression sizes than JPEG. The reconstruction of FTT cannot bring the noise.

Therefore, Quantization is the lossy stage in the JPEG coding scheme. If Quantization is too coarse, the images look "blocky" If it's too fine, more bits are needed than the final compressed data. But Quantization can be controlled with the Quality 
Factor ${ }^{(15)}$ In CCTRS, the requirements of Quantization/Dequantization must be satisfied with Fresnel transforming capability. Otherwise, the original image of CGH cannot be reconstructed using FTT.

6.3 CGH Transmitted Error and Reconstructed Image Quality There are subjective criteria to decide if a CGH and a reconstructed image are distorted or not: the observation by the eye, and the comparison between the original image and the processed image. But it is not enough to determine objectively the $\mathrm{CGH}$ quality and the reconstructed image quality. Thus we have adapted the concept of $M S E$ and $P S N R$ to determine the processed CGH quality and the reconstructed image quality in CCTRS.

According to the image processing theories ${ }^{(4)}$, the functions Eqs.(14), (15) and (16) of the compression efficiency and the distortion measure can be written. In CCTRS $R \%, M S E$ and PSNR of the processed $\mathrm{CGH}$ and the reconstructed image can be calculated, and their relationship curves can be drawn as shown in Fig.13, 14.

$$
R=\frac{S_{c}}{S_{o}}
$$

Where $R$ is Compression ratio, $S_{o}$ is defined as the size of the original $\mathrm{CGH}$ and $S_{c}$ is the size of the compressed $\mathrm{CGH}$.

$$
\begin{aligned}
& M S E=\frac{1}{L_{x} \times L_{y}} \sum_{x=1}^{x=L_{x}} \sum_{y=1}^{y=L_{y}}[f(x, y)-\bar{f}(x, y)]^{2} \\
& P S N R=10 \log _{10}\left[\frac{x_{p}^{2}}{M S E}\right](d B) \cdots \ldots \ldots \ldots
\end{aligned}
$$

Where $L_{x}, L_{y}$ are $1 \sim 512$ for CGH (or $1 \sim 1024$ for the reconstructed image of $\mathrm{CGH}) . f(x, y)$ is the image function of the original image (i.e., Fig.2, 7.), $\bar{f}(x, y)$ is the image function of the processed image. $x_{p}$ is 255 (i.e., the peak to peak value of the image data).

In terms of Table 3, 4 and Fig.13, 14, we can find out the more accuracy relationship about the original CGH, the decompressed $\mathrm{CGH}$ and the reconstructed image of CGH in CCTRS, thus we can criticize the image quality of CGH compressed and transmitted and reconstructed.

Table 3. R\% and $M S E$ and $P S N R$ about comparing the original $\mathrm{CGH}$ with the decompressed $\mathrm{CGH}$ in CCTRS.

\begin{tabular}{c|c|c|c|c}
\hline $\mathrm{q}(\mathrm{N})$ & 2 & 5 & 10 & 20 \\
\hline$R(\%)$ & 1.5303 & 3.9936 & 8.6722 & 16.5544 \\
\hline$M S E$ & 2082.7911 & 1677.7739 & 1279.0577 & 812.0562 \\
\hline$P S N R$ & 14.9444 & 15.8835 & 17.0619 & 19.0349 \\
\hline $\mathrm{q}(\mathrm{N})$ & 40 & 60 & 80 & 90 \\
\hline$R(\%)$ & 26.7317 & 34.2044 & 47.3830 & 62.7900 \\
\hline$M S E$ & 370.2311 & 187.9284 & 56.6834 & 14.8211 \\
\hline$P S N R$ & 22.4461 & 25.3909 & 30.5963 & 36.4220 \\
\hline
\end{tabular}

\section{Conclusion}

CCTRS has been established. This processing

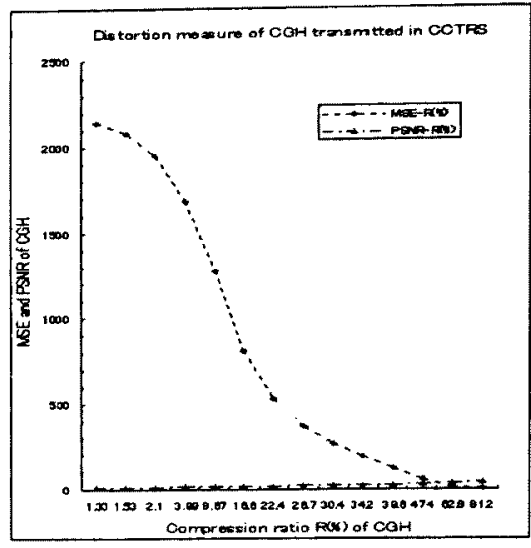

Fig. 13. Relationship curves of $R \%, M S E$ and $P S N R$ under comparing the original CGH with the decompressed CGH in JPEG baseline processing.

Table 4. R\%, MSE and PSNR about comparing the reconstructed image of original CGH with the reconstructed image of decompressed $\mathrm{CGH}$ using FTT in CCTRS.

\begin{tabular}{c|c|c|c|c}
\hline $\mathrm{q}(\mathrm{N})$ & 2 & 5 & 10 & 20 \\
\hline$R(\%)$ & 1.5303 & 3.9936 & 8.6722 & 16.5544 \\
\hline$M S E$ & 357.6149 & 31.4872 & 32.4347 & 36.1308 \\
\hline$P S N R$ & 22.5967 & 33.1495 & 33.0207 & 32.5520 \\
\hline $\mathrm{q}(\mathrm{N})$ & 40 & 60 & 80 & 90 \\
\hline$R(\%)$ & 26.7317 & 34.2044 & 47.3830 & 62.7900 \\
\hline$M S E$ & 38.4789 & 39.1351 & 38.7875 & 38.6462 \\
\hline$P S N R$ & 32.2786 & 32.2051 & 32.2439 & 32.2614 \\
\hline
\end{tabular}

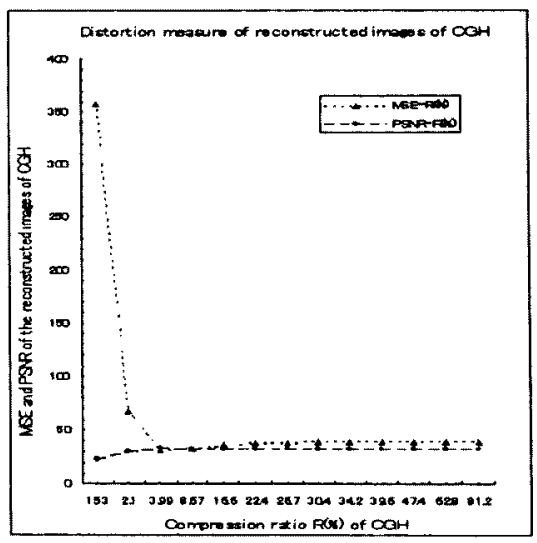

Fig. 14. Relationship curves of $R \%, M S E$ and $P S N R$ under comparing the reconstructed images of original CGH with the reconstructed images of decompressed CGH using FTT.

method of CGH compressed and transmitted and reconstructed software structure has been effectively verified by experiments. In compression and transmission experiments, the compression ratio can be achieved to $1.5303 \%$, the image information of processed CGH can be effectively reconstructed as shown in Fig.9. This system can process the bigger digital hologram (i.e., the size of displayed image is $18.07 \times 18.07\left(\mathrm{~cm}^{2}\right)$ ). In this system, the noise is caused mainly by Quantization error and FDCT algorithm is not identical slightly with 
IDCT algorithm, but we can control this error, because the Quantization and Compression Ratio can be controlled with Quality Factor (i.e., $\mathrm{q}(\mathrm{N})$ ), therefore the important higher spatial frequency components of the digital hologram can't be completely lost, consequently, encoding error may be varied smaller.

In image reconstructed experiments, according to MSE and PSNR of processed CGH, we can criticize the reconstructed image quality of processed CGH. As $10 \leq q(N) \leq 90$, MSE of reconstructed images is approximately 38 , PSNR of reconstructed images is approximately $32(\mathrm{~dB})$. In this compression range, the error value of reconstructed image of processed CGH is approximately a constant as shown in Fig.14. And the error value of reconstructed algorithm is smaller. The experiments have proved that this Fresnel transform algorithm can satisfy the requirements of image reconstructed of processed CGH.

In system experiment, we have found that the phase information of processed CGH's pixels is more important than the amplitude information of processed CGH's pixels. The amplitude information variation of CGH pixels only influences the image intensity, but the original information distribution of CGH isn't distorted. However, if the phase information of CGH pixels is more compressed (or the pixel position is more varied), it will disturb the information distribution of $\mathrm{CGH}$, thus we must choice the encoding algorithm (or compression ratio) that it will only compress the amplitude information of CGH pixels, but the phase information of CGH pixels will be lightly reduced (or it is said that the image of the processed CGH can be effectively reconstructed by Fresnel transform algorithm).

This system structure can be applied in the remote signal processing using the digital filter of CGH. Potential applications of this system include the complex facsimile equipment for digital hologram input and recording, and the coding techniques for holographic data storage systems. In the future, perhaps it may be applied in the holography movie system and the radar-guided system.

(Manuscript received October 5, 2000, revised January 8,2001 )

\section{References}

(1) G.K.Wallace, "The JPEG still picture compression standard," Commun. ACM, vol.34, pp.31-44, Apr.1991.

(2) B.V.Dasarathy, "Image data compression: Block truncation coding," Los Alamitos, Calif., IEEE Computer Society Press, 1995.

(3) V.Bhaskaran, and K.Konstantinides, "Image and video compression standards: Algorithms and Architectures," Boston, Kluwer Academic Publishers, c1995.

(4) W.D.Kou, "Digital image compression: Algorithms and Standards," Copyright (c) by Kluwer Academic Publishers, Boston/Dordrecht/London, pp.149-150, 1995

(5) R.J.Collier, C.B.Burckhardt, and L.H.Lin, "Optical holography," United Kingdom Edition Published by Academic Press, INC. (London) LTD., 1971.

(6) W.H.Lee, "Computer-Generated Holograms: Techniques and Applications," E.Wolf, Progress in Optics XVI (c) North-
Holland, 1978.

(7) K.A.Haines, and D.B.Brumm, "Holographic data reduction," Applied Optics 7, No.6, pp.1185-1189, June 1968.

(8) C.B. Burckhardt, "Information reduction in holograms for visual display," Journal of the Optical Society of America 58, No.2, pp.241-246, February 1968.

(9) L.H. Lin, "A method of hologram information reduction by spatial frequency sampling," Applied Optics 7, No.3, pp.545548, March 1968.

(10) C.B. Burckhardt, and L.H.Enloe, "Television transmission of holograms with reduced resolution requirements on the camera tube," Bell System Technical Journal 48, pp.1529-1535, May-June 1969.

(11) B.P. Hildebrand, "Hologram bandwidth reduction by spacetime multiplexing," Journal of the Optical Society of America 60, No.2, pp.259-264, February 1970.

(12) E.Wolf, "I. Bandwidth compression of optical images," Progress in optics, Volumes X, University of Rochester, N.Y., U.S.A., pp.24-30, 1972.

(13) Lucente Mark, "Holographic bandwidth compression using spatial subsampling," Optical Engineering, vol.35, No.6, pp.1529-1537, June 1996.

(14) Yoshinao Aoki, "Optical and numerical reconstruction of images from sound-wave holograms," IEEE Trans.Audio, Electroacoust, vol.AU-18, No.3, pp.258-267, 1970.

(15) J.Tsujiuchi, and K.Murata, "Optical information processing," Published in Japan, pp.328-329, 1974.

(16) Kenko Sasaki, Eichiro Tanji, Hiroshi Yoshikawa, "Data Compression for Holographic 3D Image," The journal of the Institute of Television Engineers of Japan, 48, 10, pp. 12381244(Oct. 1994).

(17) Guanglin Yang, Eiji Shimizu, "Information compressed and transmitted and reconstructed system of CGH with LOCOI image processing and Fraunhofer transforming technique," Journal of IEEJ in Japan, Vol.120-C, No.11, pp.1520-1527, November 2000.

Guanglin Yang (Non-member) is a Ph.D.student

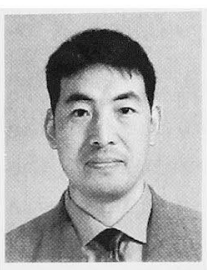
in Faculty of Electrical Engineering, Osaka City University, in Japan. He received M. E. degree from Huazhong University of Science and Technology, China, in 1993. He was a lecturer in Faculty of Automatic Control Engineering, Beijing University of Aeronautics and Astronautics, China, in 1994. His research interests mainly include the optical information processing, computer generated hologram, image processing, intelligent infrared detecting system, computer aid design automatic control system and digital control technique.

Eiji Shimizu (Member) received B.S.degree from

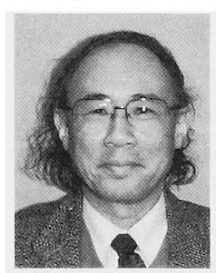

Department of Electrical Engineering in Osaka University, Japan, in 1963. He received Dr.Eng.degree from Osaka University. From 1963, he was an assistant in Department of Electrical Engineering, Osaka City University and he became a professor in 1983 . His research interests mainly include the electronic circuit, the optical electron techniques, optical information processing and neural networks. He respectively is a member of IEEE, SPIE, the Institute of Electrical Engineers of Japan, the Institute Television Engineers of Japan, and the Institute of Systems, Control and Information Engineers of Japan. 